\title{
emerald PUBLISHING
}

\section{International Journal of Workplace Health Manag}

\section{Implementation of sit-stand desks as a workplace health initiative: stakeholder views}

\begin{tabular}{|r|l|}
\hline Journal: & International Journal of Workplace Health Management \\
\hline Manuscript ID & IJWHM-02-2019-0026.R1 \\
\hline Manuscript Type: & Research Paper \\
\hline Keywords: & $\begin{array}{l}\text { Qualitative, Organizational culture, Sedentary behaviour, Physical } \\
\text { activity, Feasibility, Acceptability }\end{array}$ \\
\hline \multicolumn{2}{|l}{} \\
\hline
\end{tabular}

\section{SCHOLARONE" \\ Manuscripts}

Publisher policy allows this work to be made available in this repository. Published in Internationaل لـ Workplace Health Management by Emerald. The original publication is available at: https://doi.org/10.1108/ IJWHM-02-2019-0026. This article is deposited under the Creative Commons Attribution Non-commercial International Licence 4.0 (CC BY-NC 4.0). Any reuse is allowed in accordance with the terms outlined by the licence (https://creativecommons.org/licenses/by-nc/4.0/). To reuse the AAM for commercial purposes, permission should be sought by contacting permissions@emeraldinsight.com. 
1 Title: Implementation of sit-stand desks as a workplace health initiative: stakeholder views

\section{Abstract}

Purpose: Prolonged workplace sitting can harm employee health. Sit-stand desks are

4 a potential workplace health initiative that might reduce and break up the time office-based

5 employees spend sitting in the workplace. However, little is known about the feasibility and 6 acceptability of providing sit-stand desks. Approach: The present study sought stakeholder

7 employee views surrounding sit-stand desk implementation within two UK-based non-profit

8 organisations with open-plan offices. This paper draws on qualitative semi-structured

9 interviews with twenty-six stakeholder employees and sixty-five days of participant

10 observations. Data were analysed using thematic analysis, and organisational cultural theory

11 framed the study. Findings: Stakeholders employees' positioning of sit-stand desks as a

12 workplace health initiative reflected their perceptions of the relationship between sit-stand desk provision, employee health and organisational effectiveness. Perceptions were shaped by the nature and context of the organisation and by occupation-specific processes. Relatively fixed (e.g. organisational structure) and modifiable (e.g. selecting products compatible with the environment) factors were found to restrict and facilitate the perceived feasibility of implementing sit-stand desks. Practical implications: The findings offer several

8 recommendations for workplaces to improve stakeholder employee attitudes towards sitstand desk provision and to increase the ease and efficiency of implementation.

21 to sit-stand desk provision, this study consulted relevant stakeholders following, and

22 regarding, the sit-stand desk implementation process. 


\subsection{Introduction}

Over $74 \%$ of UK adults aged 16-64 are employed (Office for National Statistics, 2017), and a considerable policy effort is made by UK Government to support working-age adults into the workforce as reducing unemployment lessens strain on the welfare budget (e.g. Department for Work and Pensions, 2017). In addition, work can have significant benefits for the individual as it offers the chance of improved opportunities via remuneration and a sense of purpose (Institute of Health Equity, 2014). Throughout history, changing ideologies and workplace practices relating to organisational efficiency have implicated employee health and wellbeing, however contemporary approaches recognise that key to improving organisational efficiency is supporting employees' health and wellbeing (Browne, 2000). A healthy workplace has been defined by the World Health Organisation (2010) as "one in which workers and managers collaborate to use a continual improvement process to protect and promote the health, safety and wellbeing of all workers and the sustainability of the workplace" (p. 6). The political discourse positions the development of a healthy workplace as being beneficial for both individual employee health and organisational efficiency. Empirical literature supports a relationship between healthy workplaces, employee wellbeing, and organisational success (Dickson-Swift et al., 2014; Karakolis and Callaghan, 2014).

Despite the positive contribution of work to societies and individuals, work-related factors or circumstances, such as autonomy, the physical environment, and training opportunities, can be linked to poor health and wellbeing for employees (Institute of Health Equity, 2014; Smith et al., 2012). Work environments and cultures that encourage prolonged sitting and physical inactivity can harm employees' physical and mental health and wellbeing (Dunstan et al., 2012). Observational studies have consistently shown that office-workers sit for over two-thirds of the working day (e.g. Smith et al., 2015), and a recent study of over 600 desk-based workers indicated a preference for spending less time seated at work $(53.8 \%$ of the work day; Wallman-Sperlich, Chau and Froboese, 2017). In order to prevent the negative health consequences associated with prolonged workplace sitting and inactivity, and positively influence organisational success, workplaces might implement strategies that permit employees to reduce or break up prolonged workplace sitting. Sit-stand desks, i.e. height-adjustable desks allowing employees a choice of desk-based working positions, are commercially available and thus represent a potential workplace health initiative to facilitate reduced workplace sitting via regular postural change. 
Sit-stand desks are amongst the most efficacious strategies to reduce workplace sitting amongst office-based employees (Shrestha et al., 2016), and have potential to improve employee health. A recent scoping review of studies investigating the health outcomes associated with sit-stand desk use, reported positive, significant results for cardiovascular outcomes in $61 \%$ of the 14 included studies, and positive, significant results for reduced discomfort in $43 \%$ of the 22 included studies (Chambers, Robertson and Baker, 2019). However, a cross-sectional study of 680 desk-based employees found that only $16 \%$ of the employees have access to a sit-stand desk, and of these employees, only 50\% use the sit-stand function regularly (Wallman-Sperlich et al., 2017). The feasibility and acceptability of workplace sit-stand desk provision is largely influenced by the views of the employer and key stakeholders. Gilson et al. (2012) and Hadgraft et al. (2016) reported employer and practitioner hypothetical perceptions of sit-stand desks as part of exploratory studies focused on reducing workplace sitting time. However, examining the feasibility and acceptability of installing sit-stand desks alongside sit-stand desk provision is necessary to understand the potential for the wider adoption of sit-stand desks across organisations (Wijk and Mathiassen, 2011). The perceptions of organisational employees whose roles and responsibilities are implicated in sit-stand desk provision e.g. health and safety, human resources - herein described as 'stakeholder employees' - are important in understanding issues related to feasibility and acceptability.

Proponents of realistic evaluation posit that whether an intervention 'works' is not an inherent property of the intervention, but rather depends on an interaction between mechanisms - the intervention under study and stakeholders' responses - and the delivery context (Dalkin et al., 2015; Pawson and Tilley, 2004). Extent literature has highlighted that organisational cultural factors can facilitate or restrict the implementation of workplace health initiatives (Spence, 2015). Organisational culture guides how people think, feel, and act, by offering an "interpretation of an institution's history that members can use to decipher how they will be expected to behave" (Martin and Siehl, 1983, p. 52). Culture is manifest in the values and behaviours of groups, including organisations; it resides in relations between people (Alvesson, 2002, pp. 1-6) and is developed through shared and contested values; it is the context within which organisational interpretations are formed (Mannion et al., 2008).

Such and Mutrie (2016) explored the influence of organisational culture on workplace sedentary behaviour in an in-depth, qualitative study. However, there is a dearth of literature examining the role of organisational culture and context in shaping the feasibility and 
90

91

92

93

94

95

96

97

98

99

100

101

102

103

104

105

106

107

108

109

110

111

112

113

114

115

116

117

118

119

120

121

acceptability of sit-stand desk provision in workplaces. The aim of the present study was to evaluate the cultural and contextual processes influencing the feasibility and acceptability of sit-stand desk provision alongside, and in relation to, a sit-stand desk installation process.

\subsection{Methods}

\subsection{Background to the study}

(a) The wider work: This study was part of a larger project involving a 12-month pilot randomised controlled trial (RCT) and process evaluation of a multi-component workplace sit-stand desk intervention within the office-based workplaces of two non-profit organisations. The pilot RCT examined the efficacy of the intervention on reducing sitting and increasing standing and physical activity using objective activity monitors (ActivPAL3 ${ }^{\mathrm{TM}}$ and Actigraph GT3X+) at five time-points: baseline, 2-weeks, 3-months, 6-months, and 12months. There were three arms to the pilot RCT: (1) a multi-component sit-stand intervention including sit-stand desk provision, emails from managers endorsing reduced workplace sitting, and motivational interviewing delivered over the phone, (2) sit-stand desk provision only, and (3) control, i.e. no intervention. Participants in both intervention arms $(n=20)$ had a choice between two models of desk (Ergotron Workfit-A or Workfit-D, $\underline{\text { www.ergotron.com }) . ~ A t ~ t h e ~ t i m e ~ o f ~ i n s t a l l a t i o n, ~ t h e ~ p r i c e ~ o f ~ t h e ~ d e s k s ~ r a n g e d ~ b e t w e e n ~} £ 300$ (Workfit-A) and $£ 600$ (Workfit-D) depending on the retailer. All of the sit-stand desks were installed over a period of three days in the open-plan office spaces of the two participating organisations, at the place of the usual desk of the intervention participants. The desk suppliers (Workplace C) organised for an external company to complete the install, at no cost to the participating organisations, aside from assistance provided by a small number of stakeholder employees leading up to, and on, the install day(s). The protocol for the wider project is published in [author] et al. [date] and see Supplementary File 1 for an intervention delivery and evaluation timeline. Ethical approval to conduct this research study was granted by Brunel University London research ethics committee (RE45-13). All data collection took place between May 2014 and January 2016.

(b) The process evaluation: The findings from the process evaluation, underpinned by the Medical Research Council Guidelines for evaluating complex interventions (Craig et al., 2008), are reported here. Process evaluations largely focus on issues surrounding implementation and place emphasis on examining social processes to unpick how the 
122 intervention interacts with contextual factors to influence the delivery, acceptability and 123 success of the intervention (Moore et al., 2015). In the present study, a process evaluation 124 was undertaken to examine organisational attitudes related to the feasibility and acceptability 125 of sit-stand desks as a workplace health strategy. Feasibility was defined as the ease with 126 which sit-stand desk interventions can be implemented. Acceptability refers to whether 127 workplace stakeholders consider sit-stand desk interventions to be appropriate, possible and 128 permissible within their workplace context. A further characteristic of process evaluations is 129 that they work with, and aim to develop, theory to add explanatory value (Morgan-Trimmer 130 and Woods, 2016). The present study interweaved empiricism and theoretical reasoning to 131 deepen understanding of how and why people think and act and the impact this has on the 132 perceived organisational feasibility and acceptability of investing in sit-stand desks.

To gain an understanding of how workplace cultural and contextual factors underpin the feasibility and acceptability of sit-stand desk implementation, in-depth qualitative methods involving observations within the participating workplaces, and semi-structured interviews with stakeholder employees, were employed (Hawe et al., 2009). Observational methods permit the collection of data on behaviour, events and interactions as they occur within the intervention delivery context (Morgan-Trimmer and Wood, 2016). The researcher undertook a volunteer role within both workplaces to engage in observation whilst being a 'participant' (employee) within the workplace. Engaging in behaviours appropriate to the setting, rather than simply observing, can facilitate a more nuanced understanding of meanings attached to behaviours (Spradley, 1980, p. 61). Whilst observations are embedded in social settings, semi-structured interviews provide (artificial) space - outside of natural interactions - for relevant stakeholders to reflect on personal, social, work and / or occupational values, attitudes and behaviours related to sit-stand desk provision. It was proposed that combining observation and interview methods would provide the most complete and nuanced understanding of the processes influencing the feasibility and acceptability of sit-stand desk provision (Watson, 2011).

\subsection{Participating organisations and employees}

Two UK-based organisations volunteered to take part in the research, and consent was 151 obtained from senior management within both organisations. Workplace A is an established health charity. The workplace is split over several floors and is the base for over 900 employees. Workplace B is a national health-related Governmental organisation. The 
154 workplace setting was a high specification office building that was the base for over 1000 155 employees. Organisational restructuring was ongoing in both organisations throughout their 156 participation in the research. Semi-structured interviews took place with stakeholder 157 employees - whose roles and responsibilities may be implicated in sit-stand desk provision 158 from Workplaces A, B and C. Interviewees were identified via brainstorming potentially 159 relevant employees with a key contact within each organisation. This resulted in twenty-six 160 interviews being conducted with 10 stakeholders from Workplace A, 14 from Workplace B and two from Workplace C. Stakeholders' roles and responsibilities varied, however interviewees were categorised as to whether their role was operational (e.g. facilities, procurement), managerial (e.g. director, programme lead) or workplace wellbeing related (e.g. human resources advisor, workplace wellbeing champion); see Table (i). All participants received a participant information sheet and provided written informed consent prior to any data collection taking place.

\section{$\underline{2.3 \text { Data collection and analysis }}$}

Observations involved three formal phases within workplace: prior to the sit-stand desk installation, and at approximately 4 months and 10 months following sit-stand desk installation. Each phase consisted of 9-13 working days (approximately 09:00 - 17:00). The researcher observed within work spaces and departments of employee stakeholders and employees that were participating in the pilot RCT, being situated within three to four separate departments during each formal observation phase. Departments were selected across different floors, based on the type of work conducted, and according to the number of sit-stand desks within the immediate work space, to capture a range of views and behaviours. Initial, more general observations of the workplace setting and context shaped more detailed and specific observations of organisational cultural and contextual processes impacting on the sit-stand desk intervention implementation, as the data collection progressed. The researcher would make condensed notes or prompts throughout the day and expand the notes at the end of the working day (Spradley, 1980, pp. 69-70). Informal observations and interactions were also recorded throughout the duration of the interaction with the organisations. In total, 147,616 words of field notes based on the formal observation phases, and 37, 750 words of theory shaped questions and prompts relating to organisational policies, procedures, 
186 initiatives, and norms related to workplace sitting. Three pilot stakeholder interviews were 187 conducted with a Workplace Wellness Manager, a Health and Safety Manager and a senior 188 leader from a separate organisation (Brunel University London). The interview guides were 189 modified following the pilot interviews to include the addition of questions regarding sit190 stand desks, productivity, and organisational effectiveness. The guides were then tailored to 191 the interviewees' specific organisational role, and specific questions were added for some 192 stakeholders to unpick previously observed events or interactions. See supplementary file 1 193 for an example interview guide. All interviews took place approximately two months 194 following sit-stand desk installation. The interviews were 42 minutes long on average, 195 ranging from 18 to 64 minutes. All interviews were audio recorded and transcribed and 196 identifiable information was removed, including assigning all stakeholder interviewees with 197 pseudonyms. All observations and interviews were conducted by one researcher (JH). See 198 Supplementary file 2 for an illustration of the observation and stakeholder interview data 199 collection timelines, including how they fit within the wider pilot RCT work.

A thematic analysis was utilised to collectively analyse the interview and field-note 201 (observation) data across both organisations. The analysis process outlined by Braun and Clark (2013) was followed which includes: (a) data familiarisation i.e. reading and re-reading field-notes and interview transcripts, (b) the generation of initial codes, (c) interpreting codes to develop themes, and (d) reviewing, refining and defining themes. When generating and interpreting codes it was important to look beyond what participants said to understand the context within which the participants spoke and acted (Perryman, 2012) which allowed for a more process-oriented understanding of interviewees' accounts. Whilst coding was initially inductive, realistic evaluation principles also guided the conduct of the analysis. The researcher openly sought to identify the interplay between contextual factors and mechanisms on the feasibility and acceptability of sit-stand desk provision and implementation (Cruickshank, 2012, p. 14; Pawson and Tilley, 2004). One researcher (JH) reviewed and coded all raw data and another researcher (LM) independently reviewed and coded approximately $20 \%$ of the raw data; codes were compared, and discrepancies were resolved through discussions. Coding was completed using NVivo 10.0 software. One researcher (JH) interpreted the codes to develop three themes addressing the feasibility and acceptability of investing in sit-stand desks, which were reviewed, refined and agreed by all researchers $(\mathrm{JH}$,

217 LM, TK, AM). These themes, including illustrative quotes and field-note extracts provided to 218 support the analysis, are presented as findings. 


\subsection{Findings}

221

222

223

224

225

226

227

228

229

230

231

232

233

234

235

236

237

238

239

240

241

242

243

244

245

246

247

248

Three main themes related to the feasibility and acceptability of investing in sit-stand desks were generated and are presented here. The themes pertain to (1) factors influencing the perceived viability of implementing sit-stand desks, (2) organisational factors and outcomes influencing attitudes towards sit-stand desks, and (3) ethical considerations related to sit-stand desk provision.

\subsection{The viable thing to do? Factors influencing sit-stand desk implementation}

This theme describes factors influencing stakeholder employees' commitment to sitstand desk implementation, including role capacity, and organisational structure and restructuring (3.1.1), and the compatibility of sit-stand desks with the workplace environment (3.1.2) which both shape perceived sit-stand desk installation feasibility.

\subsubsection{Commitment to support sit-stand desk implementation}

Stakeholder employees' accounts revealed that the feasibility of sit-stand desk provision depends on the organisation having a dedicated staff member with capacity and commitment to lead the installation, supported by employees from relevant teams:

Where would be the natural home?... Which team would be most appropriate to deliver and support this? So, it doesn't become a Pilates ball - an expensive Pilates ball - pushed in the corner (Violet, Workplace B)

Stakeholder interviewees' accounts revealed that the perceived capacity to dedicate time to sit-stand desk installation influenced attitudes related to assisting with the delivery of the project. Katie stated that the proportion of her work hours that she dedicated to the project was "between a fifth and a quarter... it hasn't been *too* much" (Katie, Workplace A). Her commentary indicated that the incorporation of the project into her job role enabled her to prioritise the installation of the sit-stand desks. Conversely, those with less capacity were more likely to express frustration regarding installation-related tasks:

I thought 'What? I'm ordering leads? Really? How daft!... those sorts of things are seemingly petty... but for us... it's about how easy is it for an organisation to include these [sit-stand desks], well actually it is quite difficult because you need to think about all these other bits and pieces (Faye, Workplace B) 
250 project. Many employees involved in the installation could not offer sustained commitment due to fluctuating responsibilities because of ongoing restructuring processes: "Key staff in the installation process keep getting re-deployed so the contact person is changing constantly..." (Research notes, 5 Sept 2015), which also hindered communication between parties. In addition, the extent of centralisation within the organisation shaped employees' commitment to the installation of the sit-stand desks:

256

[At Workplace B] there were four or five people that needed to be making the decisions whereas, at Workplace A, it felt like once we'd got... the higher-level people on board it was just handed down.... And I guess one person is easier to deal with than multiple (Ben, Workplace C)

260 Workplace A has a less centralised structure than Workplace B, as continued and extensive involvement with technical aspects of the installation was required by fewer employees from different teams, which enabled one key employee to commit to assisting the delivery of the project. stand desks depended on the degree of formalisation. Workplace B has a formalised organisational structure; procedural necessities challenged the efficiency of the sit-stand desk planning and installation processes. For example, form-filling requirements almost led to the postponement of the installation:

Workplace B told us last week that we have to complete a RAMS (Risk and Method Statement) form... The install is due to take place next week, and if [Workplace C] are unable to complete all elements of the form by then... [team] at Workplace B have said that no work can take place on site (Research notes, $11^{\text {th }}$ Jan. 2015)

273 A higher level of formalisation at Workplace B was found to impede the efficiency with 274 which tasks connected to the sit-stand installation, a non-standard situation, could be 275 completed. Limited decision-making discretion led employees to adhere to procedures which 276 may not be wholly necessary, or indeed adequate, for sit-stand desk installation. Whereas, a 277 less formalised structure at Workplace A enabled the swift completion of tasks related to the 278 project delivery, and positively influenced employees' experiences of implementing sit-stand 279 desks within the workplace. 


\subsubsection{Sit-stand desk workplace compatibility}

281

282

283

284

285

286

287

288

289

290

291

292

293

294

295

296

297

298

299

300

301

302

303

304

305

306

307

308

309

310

The compatibility of the sit-stand desks with the office environment influenced the feasibility of sit-stand desk provision. For example, the installation of some sit-stand desks required adaptations to be made to the organisation's standard equipment, such as "sawing a curve into two desks to enable the sit-stand desk to be securely attached" (Research notes, 25 Oct. 2014). The lack of compatibility of certain aspects of the sit-stand desks with the workplace environment led to some unexpected costs for the organisation, which restricted the feasibility of the sit-stand desk installation. For example, it was necessary for the organisations to purchase longer cables to ensure that desk equipment could be powered when the desk was raised. Compatibility issues also led to unexpected time demands being placed on operational employees when sit-stand desk users required their desk to be relocated; "when I asked [Susan] if she'd had chance to have a look at moving Steph's sitstand desk to her new location, she said not yet, that she'd been "bloody busy this week to be honest" (Research notes, 20 Feb. 2015). The need to make adaptations to organisational equipment to accommodate the sit-stand desks negatively influenced interviewees' attitudes: ... it seemed as though they [Workplace C] thought that Workplace B should adapt to be able to use that desk, rather than the desk working for us ... and I think that is something that needs some thinking about, otherwise you are going to start off with already some negative thinking...” (Faye, Workplace B)

This theme revealed that organisational contextual factors can impede or facilitate the sit-stand desk implementation process, and consequently, stakeholder employees' perceptions of the feasibility of sit-stand desk provision. The viability of sit-stand desk provision is likely to vary between organisations depending on the organisations' context, structure, and physical workplace environment.

\subsection{The smart thing to do? Organisational factors and outcomes}

This theme links sit-stand desk provision to employee health and organisational outcomes. Firstly, the perceived organisational benefits of investing in sit-stand desks are described (3.2.1). Organisational factors influencing perceptions of the importance of supporting employee health (3.2.2) and the relationship between supporting employee health via sit-stand desk provision and organisational outcomes (3.2.3) are presented.

\subsubsection{Organisational benefits of investing in sit-stand desks}


311 Employees voiced that the pursuit of organisational success guides business 312 operations and decisions, including whether to invest in employee health initiatives. For 313 example, Jill reasoned that "if our y'know, reason for being is to help improve the lives of 314 everyone living with [health condition] then if we can i-you have improved physical health... 315 you will also be more productive. If you are absent from work, then obviously, you are not 316 productive. But even if you are in work and you're not well then you will be less productive 317 than someone else who is feeling great (Finn, Workplace B)

318 Interviewees perceived that employees seek out job opportunities that offer benefits including 319 staff wellbeing initiatives: ...but you know actually bringing it into the workplace and having specially adapted desks, you know I was pleased that we were doing something that felt a little bit ahead of maybe our competitors (Jayne, Workplace A)

323 Sit-stand desks were positioned as an aspect of competitive advantage. Employees also 324 identified a need for the organisation to 'practice what they preach':

We need to make sure that we are exemplar health and wellbeing employers for our own staff, because otherwise why on earth would anybody listen to us when we say this is best practice... (Richard, Workplace B)

The organisations' external programmes of work are oriented around improving peoples' health and wellbeing. Thus, interviewees perceived that it is necessary to support staff health and wellbeing internally, to appear a credible source of information to stakeholders including the public. The health-focused nature of the participating organisations garnered additional support for investing in sit-stand desks. Sit-stand desks were described as a visible and forward-thinking representation of the organisations' commitment to developing a healthy workplace.

\subsubsection{Organisational and occupational values influencing attitudes towards sit-stand desks} organisational (health) culture and employees' occupational identities. The health-related nature of both organisations engendered taken-for-granted assumptions amongst interviewees about the importance of health and wellbeing: 
We've got the tag line now, of protecting and improving the nation's health. It's literally on every email you send... so it should be in your eye line *laughs* every single day... this is who we are (Annabel, Workplace B)

Valuing health was embedded throughout the organisations and reflected in organisational materials, which led to a shared understanding amongst employees that "healthy living are [sic] generally better for you and you know, give you better outcomes" (Steph, Workplace A). For employees in health-focused roles, e.g. human resources, their occupational identities were consistent with and reinforced the dominant organisational values.

However, some interviewees that were employed in operational, non-health-focused occupations, for example estates and facilities, held negative perceptions regarding sit-stand desks. For example, Dorothy questioned "what benefit there is, health wise? Standing for long periods is not good, because you get stagnation of blood... varicose veins" (Dorothy, Workplace B). In addition, some interviewees viewed sit-stand desks as a luxury rather than as a preventative health measure that should be available to all employees:

I think it's quite different to something like, you know a laptop or an iPhone, which is essential to conducting our business... [but] certainly if there was a physical issue or health reason.... we would be supportive (Tarak, Workplace B)

Employee health and wellbeing are not always prioritised within non-health focused occupations. This analysis indicates that organisational cultural contradictions can arise when the occupational values of employees responsible for the management of office equipment are not consistent with those of the organisation:

She [health-focused Workplace B employee] vented her frustrations regarding the current procedure, saying 'we have to have a [health] problem, a symptom, a justification to request a sit-stand desk at Workplace $B$, which goes against all the work we are trying to do nationally' (Research notes, 19 th Sept. 2014)

Seated desks as standard office equipment were symbolised by employees with health-related occupations as inadequately investing in the health of their staff, which undermines the organisations' the dominant cultural value of preventative health.

\subsubsection{Financial implications of investing in sit-stand desks}


Interviewees identified that evidence of cost-effectiveness and ROI would be required to justify any future financial investment in sit-stand desks:

Interviewees expressed concern regarding a potential negative reaction of employees and the public to organisational spend on sit-stand desks. At Workplace B, this was influenced by the organisational economic climate. The governmental stipulation to reduce organisational spending incited restructuring, job losses and increased workload at Workplace B.

Organisational conditions fostered low need satisfaction, which engendered negative attitudes towards formal employee health provision amongst employees:

Employees see these [employee health] initiatives as 'add-ons', 'not getting at the root cause', 'putting over a plaster' - so not solving the problems inherent within the organisation - long hours, email culture, constant restructuring and job insecurity (Researcher notes, Workplace B, 25 th Nov. 2015)

Spending money on employee health initiatives was perceived by some employees as a feeble attempt to minimise negative impacts on staff wellbeing caused by organisational conditions, which was recognised by interviewees:

It would be quite difficult to weigh up if you went for a carte blanche and spent thousands and thousands because... we're cutting staff.... so, it would be a delicate balance for senior management to promote that.... in the current climate (Ivy, Workplace B)

Concern that substantial expenditure on sit-stand desks might exacerbate negative feelings amongst employees towards employee health initiatives engendered a perception that spending money on sit-stand desks is not justifiable. Interviewees expressed concern that expenditure on sit-stand desks might negatively influence the organisations' image:

You'd have to be accountable for spending public funds... and certainly as a taxpayer I would be pretty annoyed (Dorothy, Workplace B)

This theme indicates that the 'healthy workplace' rationale for investing in sit-stand desks is strengthened due to the health-focused nature of the organisations, as appearing a 'credible source' is unlikely to be linked to organisational success for non-health-focused 
organisations. However, views differ between individual employees due - in part - to

400 differing occupational identities, and financial concerns are likely exacerbated in public or charitable organisations compared to private organisations. These findings highlight that organisational contextual factors factor into, and complicate, the relationship between employee health initiatives and organisational effectiveness, and influence stakeholder employees' attitudes towards sit-stand desk provision.

3.3 The right thing to do? Challenging the ethics of the healthy workplace model

406

407

408

409

410

411

412

413

414

415

416

417

418

419

420

421

422

423

424

425

426

427

428

429

The data in this theme considers issues surrounding the corporate social responsibility of organisations with office-based workplaces to provide sit-stand desks for their employees.

Interviewees spoke of an ethical responsibility of the employer for supporting employee health, in part due to the nature of their organisation:

I think a responsible employer... their core philosophy has to be look[ing] after their employees... we are [Workplace B]... it would be wrong... if we were generating overworked, stressed out people just about to break (Bridget, Workplace B)

The data highlights a view that employers have a responsibility not to contribute to reduced employee health and wellbeing via physical or psychosocial working conditions. Positioning the provision of sit-stand desks as a responsibility of office-based organisations, to offset the health risks incited by prolonged workplace sitting, increases the acceptability of sit-stand desks within such organisations. However, deeper analysis of interview and observation data illustrate that employees would not expect their organisation to support their health without there also being benefits for the organisation. For example, Cath commented that sit-stand desks are "the right thing to do" yet later remarked that "businesses are businesses, and they look at the bottom line" (Cath, Workplace A).

Interviewees recognised that employee health initiatives can be used in ethically dubious ways. For example, Finn described attempts of "tech[nology] companies" to, in his view, overtly regulate the behaviour of their employees. Discussing a recent visit to one such technology company, Finn described how "everything you need is within the building, so you never need to leave. They have a gym, they have breakfast, they have everything. Food all day basically. So, they keep them there.... terrible" (Finn, Workplace B, workplace wellbeing stakeholder). There was a feeling amongst interviewees that imposing health behaviours on employees represents an abuse of the organisations' power. Regarding employee eating 
430 habits, Jayne remarked "why should we be prescriptive with people about what they do...

431 what they put in their mouth... we haven't got a right to do that" (Jayne, Workplace A).

432 Interviewees expressed a belief that the organisation should be supportive of health by not

\subsection{Discussion}

This study examined the feasibility and acceptability of implementing sit-stand desks in office-based workplaces by conducting observations and interviewing key stakeholders within two workplaces taking part in a pilot RCT of a workplace sit-stand desk intervention. Factors related to the sit-stand desk implementation process, perceived organisational benefits and costs, and ethical issues linked to supporting employee health were illustrated as being instrumental in shaping views regarding sit-stand desk provision.

\section{Sit-stand desk provision and implementation: mediating causal mechanisms}

\section{Mediating mechanisms relate to components of an intervention and how it is} implemented that have an influence on outcomes, e.g. how sit-stand desk provision is perceived, and the ease of implementation (Dalkin et al., 2015). Whilst the two workplaces that delivered the intervention were both health-focused, non-profit organisations, and mediating mechanisms interact with context to influence outcomes, the analysis pointed to modifiable factors that might influence the feasibility and acceptability of sit-stand desk provision and implementation across organisations more generally.

Positive attitudes related to providing sit-stand desks were consistent with the healthy workplace discourse - which posits that reduced absenteeism and presenteeism, and increased work motivation, increase productivity via improved employee health and wellbeing 
461 (Wadsworth et al., 2010) - as a rationale for investing in sit-stand desks. Indeed, extant

462 literature is supportive of a relationship between using a sit-stand desk and employee health

463 benefits, including improved musculoskeletal comfort and cardiovascular outcomes

464 (Chambers, Robertson and Baker, 2019). The pursuit of productivity and organisational

465 success is a taken-for-granted priority, which guides organisational operations (Such and

466 Mutrie, 2016). Thus, positioning sit-stand desk provision as a strategy to increase

467 organisational effectiveness - via improved employee health - is likely to increase

468 organisations' receptibility towards supplying sit-stand desks for employees. Additionally,

469 highlighting the consistency between sit-stand desk provision and the occupational values of

470 key organisational decision-makers is likely to increase acceptability. However, the findings

471 indicate that employees are wary of organisations supporting employee health for underlying

472 corporate motives; such motives question the positioning of workplace health initiatives as

473 socially responsible (Holmqvist et al., 2009), as they represent a means for the organisation

474 to - wittingly or unwittingly - manipulate and control employees' health-related attitudes and

475 behaviour (Vander Schee, 2008). Offering employees the choice of a sit-stand desk or seated

476 desk was considered an appropriate strategy, as interviewees felt that such an approach

477 increases employees’ capacity for agency over their health behaviours at work. Such a

478 strategy might increase the acceptability of sit-stand desk provision amongst employees and

479 across organisations more broadly. However, placing value on employee health can lead to

480 the development of a normative power which may compel individuals to partake in healthy

481 behaviours and workplace initiatives (Zoller, 2003), even when participation is construed as

482 voluntary by the organisation, given the moral connotations of, and virtues (e.g. discipline,

483 self-responsibility) connected to, health behaviours (Verdonk et al., 2010).

The analysis identified various modifiable factors related to the implementation process that influenced views related the feasibility and acceptability of sit-stand desk

486 implementation. Factors that might improve the ease of implementation of sit-stand desks

487 include: designating a lead employee with overall responsibility for sit-stand desk provision,

488 assembling small project support team consisting of employees from key relevant

489 departments including (but not limited to) health and safety, internal communications,

490 facilities, and human resources, a clear internal communication plan, having a budget for

491 unexpected costs, and choosing models of sit-stand desks that are compatible with the

492 workplace environment.

493 Sit-stand desk provision and implementation: moderating causal mechanisms (context) 
The analysis unpicked various factors linked to the culture and context of the

495 participating organisations that influenced stakeholder views related to sit-stand desk

496 provision and implementation. For example, the findings reveal nuanced and context-specific

497 processes by which supporting employee health might be connected to organisational

498 outcomes. The health-focused nature of the participating organisations led to a perception

499 that supporting employee health would enhance the organisations' credibility. Conversely,

500 spending charity or public money on sit-stand desks might damage the organisations'

501 reputation; there can be increased scrutiny of spend, and a greater sense of accountability to

502 external stakeholders, in non-profit organisations compared to private organisations

503 (Dhanani, 2009). Financial concerns linked with the current dearth of ROI evidence (Buckley

504 et al., 2015) limited the possibility of wider adoption of sit-stand desks within the

505 participating organisations. The healthy workplace model should recognise how complex

506 organisational contextual factors such as the nature of the organisation and employees'

507 occupations can influence the processes that underpin, and the type and strength of the

508 relationship between employee health initiatives and organisational success.

509 Organisational contextual factors that impeded or facilitated the sit-stand desk

510 implementation process were identified. For example, higher organisational structural

511 formalisation - which refers to the presence of rules, policies and procedures that stipulate

512 organisational operations and decisions (Pertusa-Ortega, Zaragoza-Sáez, \& Claver-Cortés,

513 2010) - restricted implementation. The feasibility of initial sit-stand desk installation may be

514 reduced in organisations with highly formalised structures as rules and processes designed to

515 increase efficiency can contribute to inefficiency in new or non-standard situations (Bozeman

516 and Scott, 1996, p. 3). However, routine sit-stand desk provision might trigger the adaptation

517 of current procedures or development of new policies suited to the task to increase the

518 efficiency of sit-stand desk implementation (DeHart-Davis et al., 2013).

519 Strengths and limitations

520 Whilst extant literature has investigated managers' and practitioners' attitudes

521 towards hypothetical sit-stand desk provision (Gilson et al., 2012; Hadgraft et al., 2016) this

522 study consulted relevant stakeholders following, and regarding, the sit-stand desk

523 implementation process. Conducting observations was particularly valuable for examining

524 organisational culture and contextualising interview data, as it involves the collection of data

525 within the naturalistic setting and enables direct access to organisational processes and 
526 employee interactions (Morgan-Trimmer and Wood, 2016). However, the generalisability of

527 the findings is uncertain as they are based on a study of only two workplaces. Investigation

528 into organisational contextual factors that impede and restrict the sit-stand desk

529 implementation process within varied organisational settings would help strengthen and

530 extend the evidence generated in this study. A detailed map of relevant organisational

531 contextual factors and the processes that link them to the ease of implementation would

532 permit an assessment of how feasible sit-stand desk implementation is likely to be for a given

533 organisation. This study contributed to methodological advancement of approaches to

534 conducting process evaluations of workplace sit-stand desk interventions by combining

535 interviews with participant observation.

536 Conclusion

537 The present study sought stakeholder employee views of sit-stand desks as a

538 workplace health initiative alongside the implementation of a sit-stand desk intervention

539 within two UK office-based workplaces. This approach permitted the examination of

540 organisational contextual and structural factors that influence sit-stand desk implementation

541 feasibility and acceptability, which may assist organisations in selecting appropriate

542 workplace health interventions and improve the ease and acceptability of sit-stand desk

543 provision; see Figure (ii) for workplace recommendations. Identified modifiable factors

544 influencing feasibility and acceptability - such as developing an appropriate communication

545 strategy - can assist organisations in improving the process of implementing sit-stand desks.

546 However, various factors related to the organisational culture and context were found to

547 moderate attitudes related to the sit-stand desk provision and implementation. Given the

548 relatively fixed nature of organisations, employers should carefully consider the suitability of

549 sit-stand desks as a workplace health strategy within the context of their organisation - taking

550 into consideration the moderating factors influencing feasibility and acceptability identified

551 in this study linked to the nature, structure, and values of organisations. 


\section{References}

553

554

Alvesson, M. (2002). Understanding organizational culture. London: SAGE.

555

556

Bozeman, B., and Scott, P. (1996). "Bureaucratic red tape and formalization: Untangling 557 conceptual knots", The American Review of Public Administration, 26(1), 1-17. doi:

558 $10.1177 / 027507409602600101$

559

560

Braun, V., and Clarke, V. (2013). Successful qualitative research: A practical guide for 561 beginners. London: SAGE.

562

563

Browne, J. H. (2000). "Benchmarking HRM practices in healthy work organizations", 564 American Business Review, Vol. 18 No. 2, pp. 54-61.

565

566

Buckley, J. P., Hedge, A., Yates, T., Copeland, R. J., Loosemore, M., Hamer, M., . . .

567

Dunstan, D. W. (2015). “The sedentary office: A growing case for change towards better health and productivity. Expert statement commissioned by Public Health England and the Active Working Community Interest Company”, British Journal of Sports Medicine, Vol. 49 No. 21, pp. 1357-1362.

571

572 Chambers, A. J., Robertson, M. M., and Baker, N. A. (2019). "The effect of sit-stand desks 573 on office worker behavioral and health outcomes: A scoping review", Applied Ergonomics, 574 Vol. 78, pp. 37-53.

576 Craig, P., Dieppe, P., Macintyre, S., Michie, S., Nazareth, I., and Petticrew, M. (2008).

577 "Developing and evaluating complex interventions: The new Medical Research Council 578 guidance”, British Medical Journal, Vol. 337, pp. 185-202.

580 Cruickshank, J. (2012). "Positioning positivism, critical realism and social constructionism in 581 the health sciences: A philosophical orientation”, Nursing Inquiry, Vol. 19, pp. 71-82. 
583 Dalkin, S. M., Greenhalgh, J., Jones, D., Cunningham, B., and Lhussier, M. (2015). "What's

584 in a mechanism? Development of a key concept in realist evaluation", Implementation

585 Science, Vol. 10 No. 49.

586

587 DeHart-Davis, L., Chen, J., and Little, T. D. (2013). "Written versus unwritten rules: The role 588 of rule formalization in green tape", International Public Management Journal, Vol. 16 No.

5893 , pp. 331-356.

590

591 Dhanani, A. (2009). “Accountability of UK charities", Public Money and Management, Vol. 59229 No. 3, pp. 183-190.

593

594 Dickson-Swift, V., Fox, C., Marshall, K., Welch, N., and Willis, J. (2014). "What really 595 improves employee health and wellbeing", International Journal of Workplace Health 596 Management, Vol. 7 No. 3, pp. $138-155$.

597

598

Dunstan, D.W., Howard, B., Healy, G.N., and Owen, N. (2012). "Too much sitting - A 599 health hazard", Diabetes Research and Clinical Practice, Vol. 97, No. 3, pp. 368 - 376. 600

601 Gilson, N., Straker, L., and Parry, S. (2012). “Occupational sitting: Practitioner perceptions 602 of health risks, intervention strategies and influences", Health Promotion Journal of 603 Australia, Vol. 23 No. 3, pp. 208-212. 604 605

Griffeth, R. W., Hom, P. W., and Gaertner, S. (2000). "A meta-analysis of antecedents and 606 correlates of employee turnover: Update, moderator tests, and research implications for the 607 next millennium", Journal of Management, Vol. 26 No. 3, pp. 463-488. 608 609 Hadgraft, N., Brakenridge, C., LaMontagne, A., Fjeldsoe, B., Lynch, B., Dunstan, D., . . . 610 Lawler, S. (2016). "Feasibility and acceptability of reducing workplace sitting time: A 611 qualitative study with Australian office workers", BMC Public Health, Vol. 16 No. 933. 612

613 Hawe, P., Shiell, A., and Riley, T. (2009). Theorising interventions as events in systems. 614 American Journal of Community Psychology, Vol. 43 No. 3, pp. 267-276. 
616 Holmqvist, M., Företagsekonomiska, I., Stockholms, U., and Samhällsvetenskapliga, F. 617 (2009). "Corporate social responsibility as corporate social control: The case of work-site 618 health promotion", Scandinavian Journal of Management, Vol. 25 No. 1, pp. 68-72.

620 Institute of Health Equity (2014). Local action on health inequalities: Increasing employment 621 opportunities and improving workplace health. Available from:

622 https://www.gov.uk/government/publications/local-action-on-health-inequalities-evidence623 papers

624

Karakolis, T., and Callaghan, J. P. (2014). "The impact of sit-stand office workstations on 626 worker discomfort and productivity: A review”, Applied Ergonomics, Vol. 45 No. 3, pp. 799627806.

628

629 Mannion, R., Davies, H., Tobias, J., Scott, T., Bower, P., Whalley, D., . . McMurray, R. 630 (2008). Measuring and assessing organisational culture in the NHS (OC1). NCCSDO. 631

Martin, J., and Siehl, C. (1983). “Organizational culture and counterculture: An uneasy 634 symbiosis", Organizational Dynamics, Vol. 12 No. 2, pp. 52-64.

Martz, W. (2013). "Evaluating organizational performance: Rational, natural, and open 636 637 system models", American Journal of Evaluation, Vol. 34 No. 3, pp. 385-401.

Morgan-Trimmer, S., and Wood, F. (2016). "Ethnographic methods for process evaluations 644

Moore, G.F., Audrey, S., Barker, M., Bond, L., Bonell, C., Hardeman, W., . . B Baird, J. 647

648 Pawson, R., and Tilley, N. (2004). Realist evaluation: The magenta book guidance notes. 649 London: Cabinet Office. 
651 Perryman, J. (2012). "Discourse analysis". In A.R.J. Briggs, M. Coleman and M. Morrison

652 (Eds.), Research methods in educational leadership and management, London: SAGE

653 Publications Ltd, pp. 309-322.

654

655 Pertusa-Ortega, E. M., Zaragoza-Sáez, P., and Claver-Cortés, E. (2010). "Can formalization, 656 complexity, and centralization influence knowledge performance?", Journal of Business

657 Research, Vol. 63 No. 3, pp. 310-320.

658

659 Shrestha, N., Kukkonen-Harjula, K. T., Verbeek, J. H., Ijaz, S., Hermans, V., and Bhaumik, 660 S. (2016). "Workplace interventions for reducing sitting at work", The Cochrane Database of 661 Systematic Reviews, Vol. 3.

662

663 Smith, L., Ekelund, U., and Hamer, M. (2015). "The potential yield of non-exercise physical 664 activity energy expenditure in public health", Sports Medicine, Vol. 45 No. 4, pp. 449-452. 665

666 Smith, S., Makrides, L., Lebel, F.S., Allt, J., Montgomerie, D., Farquharson, J., . . .

667 Szpilfogel, C. (2012). "The healthy lifeworks project: the role of organisational health in the 668 personal health of employees", International Journal of Workplace Health Management, Vol. 669 5, No. 3, pp. $194-209$.

670

671 Spence, G. B. (2015). "Workplace wellbeing programs: If you build it they may NOT come...because it's not what they really need!", International Journal of Wellbeing, Vol. 5 No. 2, pp. 109-124.

674

Spradley, J. P. (1980). Participant observation. London, Harcourt Brace Jovanovich. 676

677 Such, E., and Mutrie, N. (2016). "Using organisational cultural theory to understand 678 workplace interventions to reduce sedentary time", International Journal of Health Promotion and Education, Vol. 55 No. 1, pp. 18-29.

681 The Department of Work and Pensions (2017). Work, health and disability green paper:

682 improving lives. Available from: https://www.gov.uk/government/consultations/work-health683 and-disability-improving-lives. 
685 Vander Schee, C. (2008). "The politics of health as a school-sponsored ethic: Foucault, 686 neoliberalism, and the unhealthy employee", Educational Policy, Vol. 22 No. 6, pp. 854-874. 687 688 689 690

Verdonk, P., Seesing, H., and de Rijk, A. (2010). "Doing masculinity, not doing health? A qualitative study among Dutch male employees about health beliefs and workplace physical activity", BMC Public Health, Vol. 10 No. 712.

691

692

Wadsworth, E. J., Chaplin, K. S., Allen, P. H., and Smith, A. P. (2010). "What is a good job? 693 Current perspectives on work and improved health and well-being”, Open Occupational 694 Health and Safety Journal, Vol. 2 No. 1, pp. 9-15.

695

696

Wallmann-Sperlich, B., Bipp, T., Bucksch, J., and Froboese, I. (2017). "Who uses height697 adjustable desks?-Sociodemographic, health-related, and psycho-social variables of regular 698 users", International Journal of Behavioral Nutrition and Physical Activity, Vol. 14 No. 26.

699

Wallmann-Sperlich, B., Chau, J. Y., and Froboese, I. (2017). "Self-reported actual and 701 desired proportion of sitting, standing, walking and physically demanding tasks of office 702 employees in the workplace setting: do they fit together?", BMC Research Notes, Vol. 10 No. 703 504.

704

705

706

Watson, T.J. (2011). "Ethnography, reality, and truth: the vital need for studies of 'how things work'in organizations and management", Journal of Management studies, Vol. 40 No.1, pp.202-217.

708

Wijk, K., and Mathiassen, S. E. (2011). "Explicit and implicit theories of change when 710 designing and implementing preventive ergonomics interventions-A systematic literature review", Scandinavian Journal of Work, Environment and Health, Vol. 37 No. 5, pp. 363712 375.

713

World Health Organisation (2010). Healthy workplaces: A model for action: For employers, workers, policy-makers and practitioners. WHO. 
717 Zoller, H. M. (2003). "Working out managerialism in workplace health promotion", 718 Management Communication Quarterly, Vol. 17 No. 2, pp. 171-205. 


\begin{tabular}{|c|c|c|c|}
\hline Simon & Director (Corporate Resources) & Senior / middle management & $\mathrm{A}$ \\
\hline Jade & Programme Lead & Senior / middle management & $\mathrm{A}$ \\
\hline Cath & Programme Lead & Senior / middle management & A \\
\hline Craig & Consultant (Public Health) & Senior / middle management & $\mathrm{B}$ \\
\hline Samantha & Human Resources Advisor & Workplace wellbeing & A \\
\hline Jill & Human Resources Manager & Workplace wellbeing & $\mathrm{A}$ \\
\hline Violet & Communications officer & Workplace wellbeing & $\mathrm{B}$ \\
\hline Fiona & Equality and Diversity Lead & Workplace wellbeing & $\mathrm{B}$ \\
\hline Faye & Staff Wellbeing Lead & Workplace wellbeing & $\mathrm{B}$ \\
\hline Finn & Staff Wellbeing Lead & Workplace wellbeing & $\mathrm{B}$ \\
\hline Annabel & Workplace Wellbeing champion & Workplace wellbeing & $\mathrm{B}$ \\
\hline Chloe & Research Manager & Workplace wellbeing & $\mathrm{C}$ \\
\hline Jim & Facilities Manager & Operational & A \\
\hline David & Health and Safety Advisor & Operational & A \\
\hline Katie & Project Officer & Operational & A \\
\hline Alexandra & $\begin{array}{l}\text { Business and Performance } \\
\text { Manager }\end{array}$ & Operational & $\mathrm{B}$ \\
\hline Ivy & Estates and Facilities Manager & Operational & $\mathrm{B}$ \\
\hline Barbara & Procurement Manager & Operational & $\mathrm{B}$ \\
\hline Aiden & Procurement Manager & Operational & $\mathrm{B}$ \\
\hline Harry & Project Sponsor (Estates) & Operational & $\mathrm{B}$ \\
\hline Ben & Account Manager & Operational & $\mathrm{C}$ \\
\hline Richard & Human Resource Director & $\begin{array}{l}\text { Senior / middle management, } \\
\text { Workplace wellbeing }\end{array}$ & A \\
\hline Olivia & $\begin{array}{l}\text { Consultant (Occupational } \\
\text { Health) }\end{array}$ & $\begin{array}{l}\text { Senior / middle management, } \\
\text { Workplace wellbeing }\end{array}$ & B \\
\hline Jayne & Human Resources Director & $\begin{array}{l}\text { Senior / middle management, } \\
\text { Workplace wellbeing }\end{array}$ & \\
\hline Drew & Head of Procurement & $\begin{array}{l}\text { Senior / middle management, } \\
\text { Operational }\end{array}$ & \\
\hline Tarak & $\begin{array}{l}\text { Deputy Director (Corporate } \\
\text { Risk) }\end{array}$ & $\begin{array}{l}\text { Senior / middle management, } \\
\text { Operational }\end{array}$ & B \\
\hline
\end{tabular}




\section{SIT-STAND DESKS AS AN EMPLOYEE HEALTH STRATEGY:} RECOMMENDATIONS FOR WORKPLACES

\section{To increase acceptability of sit-stand desk provision:}

- Employees in health-related roles should champion sit-stand desks to convince employees in non-health-related roles of the benefits of supporting employee health

- Employees in health-related roles (for example, human resources) should work alongside facilities employees in the procurement of desk-based equipment, to increase the likelihood of employee health considerations being incorporated into desk selection

- Develop a communication strategy to inform employees and external stakeholders of why sit-stand desks are a positive investment, to reduce the likelihood of reputational damage and negative employee attitudes

- Consider potential cost-saving options, e.g. investing when a refurbishment is required, investing in a small number of sit-stand desks, seeking sit-stand desk donations or explore external funding avenues

- If only investing in a small number of sit-stand desks, consider which employees should receive them. Provide sit-stand desks for employees that want one, focusing on alternate strategies for reducing sitting for employees who express negative attitudes towards sit-stand desks

\section{To increase the ease of sit-stand desk implementation:}

- Provide leadership by a relevant department; and form a project team to support the implementation, involving employees from other relevant departments including health and safety, IT, facilities, communications, etc. The project team should be small to facilitate open and close communication

- Incorporate sit-stand desk provision into the job role of at least one employee, to increase capacity for investing time into sit-stand desk provision

- Allocate an agreed budget for sit-stand desk provision and monitor budget over time

- Extensively research sit-stand desk options and select models that are compatible with the workplace environment, and conduct a site survey with potential sit-stand desk suppliers to recognise the specific requirements of the organisation for successfully installing sit-stand desks

- Develop organisation-specific policies and processes for sit-stand desk provision, based on the organisational context and early experiences of sit-stand desk provision, to improve the efficiency of sit-stand desk provision over time 


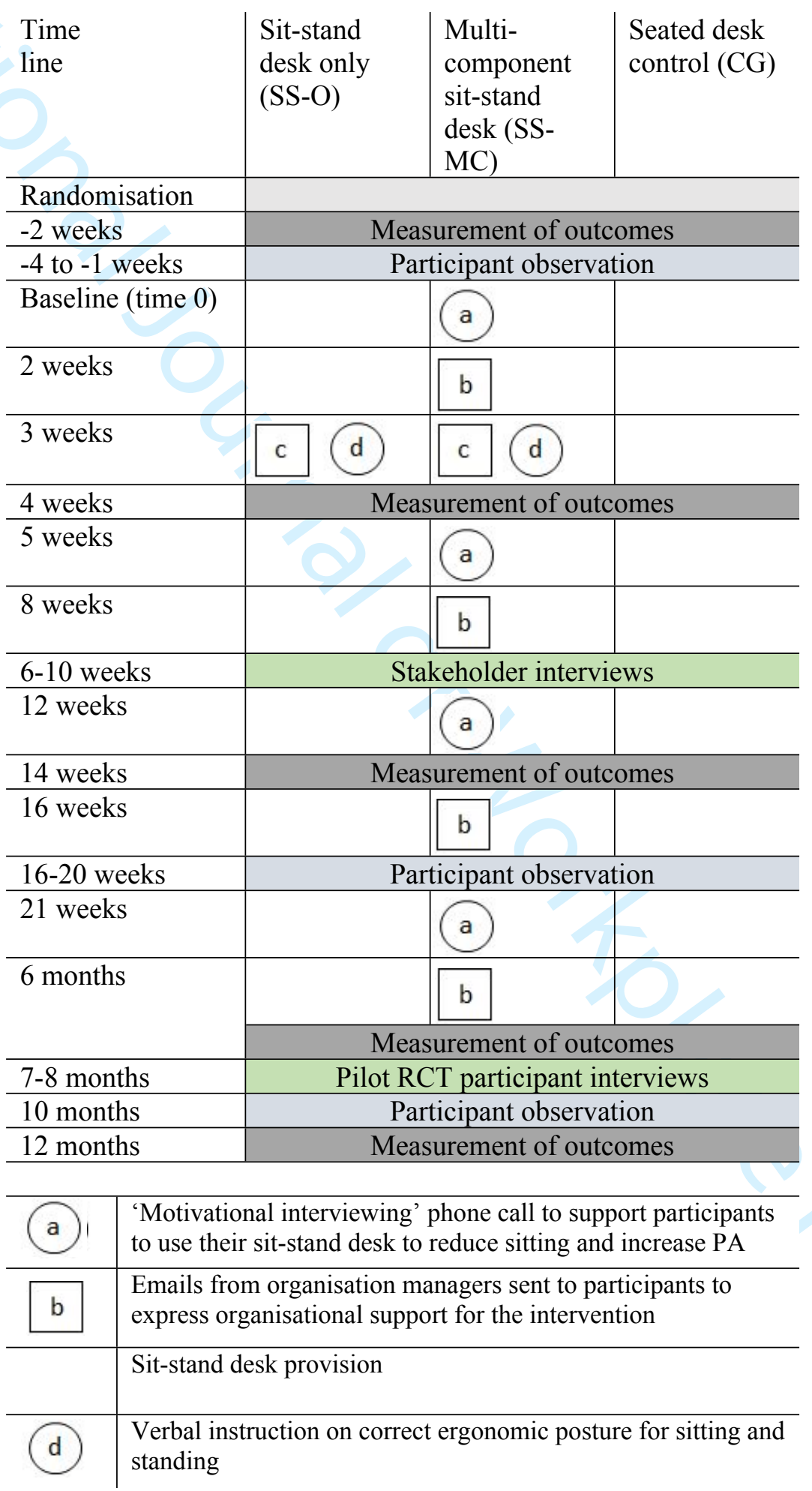




\section{Example stakeholder interview guide (health and safety staff member)}

Part A: Job role

- What is your role within the organisation?

o (PROMPTS: how does it fit into organisation aims, what do you do day-to-day)

- How does your job role relate to the sit-stand project?

- (PROMPTS: Do you see it as having an impact on your work stream? Do you have any specific roles or responsibilities as part of this project?

Part B: Understanding the culture of the organisation in relation to sit-stand desks and workplace health

- Are there any H\&S policies/training related to sitting, standing and moving?

○ (PROMPTS: restricting or encouraging movement/activity)

- Do you feel that employees have opportunities to reduce sitting and increase activity in the workplace?

- Does the sit-stand desk intervention fit with [Workplace $A^{\prime}$ s] health, safety and wellbeing approach?

- (PROMPTS: Will any amendments need to be made to current policy / guidelines to accommodate sit-stand working? Have you learnt anything so far from your experience with sit-stand working? Does the management style/priorities of senior leaders influence your role in delivering health, safety and wellbeing programmes?)

Part C: Responsibility of the organisation

- Do you feel there is a desire within the organisation to promote health of employees?

- (PROMPTS: How much of a priority is it? Does [Workplace A] have a responsibility to employees to promote their health? If so, why? (e.g. organisational benefits, nature of organisation, ethically) What about reducing sitting specifically?)

Part D: Planning and implementation of sit-stand project

- What do you think went well in the planning phases of this project? What could we have done better? How could we have done it better?

- Can you anticipate any challenges over the next year?

- What do you think would need to happen for [Workplace A] to consider a wider adoption of sit-stand desks?

O (PROMPTS: Would there be any special considerations from a health and safety perspective?) 